\title{
Young vegetarians and omnivores
}

\section{Dietary habits and other health-related aspects}

\section{Christel Larsson}

The general aim of the present thesis was to describe the prevalence of vegetarians $(1,2)$, their food habits (2), lifestyle characteristics (3) and nutrient intake, and the nutritional status of young vegetarians and omnivores $(4,5)$.

The prevalence of vegetarians was investigated among 2041 students by classifying people as vegetarians according to self-reported dietary intake (2). Vegetarians were defined as people eating vegan food and lacto-vegetarian, lacto-ovo-vegetarian or semi-vegetarian food. The prevalence of 15-year-old vegetarians in Umeå, Sweden, was $15.6 \%$ in 1996, compared with $4.8 \%$ in Stockholm, Sweden and $3.8 \%$ in Bergen, Norway. In Umeå, lacto(-ovo)-vegetarians (8.8\%), including lacto-vegetarians and lacto-ovo-vegetarians, were the most common type of vegetarians, but in Stockholm and Bergen semi-vegetarians were more common $(2.6 \%$ and $2.3 \%$, respectively). There was a higher percentage of females among vegetarians in Umeå and Stockholm $(p<0.01)$ but no gender difference was found in Bergen. Assuming that students (16-20 years old) eating vegetarian food at school lunch really are vegetarians, the prevalence of vegetarians in Sweden was 5.0\% (1). Two per cent were eating semi-vegetarian food, $2.9 \%$ lacto-ovo-vegetarian food and $0.1 \%$ vegan food. There was a higher occurrence of students eating vegetarian school lunches in the town of Umeå $(11.4 \%)$ than in Sweden in general.

The food habits and lifestyle characteristics of 2041 adolescents (15 years old) was investigated by questionnaire in 1996 (2). Besides eating less meat and other animal products, the female vegetarians less often consumed potatoes, soft drinks, chips/ peanuts, cakes/cookies, and fried and barbecued food, while more often consuming rice/pasta, vegetables, coffee, tea, microwave-heated food and dietary supplements than did the female omnivores. Even though female vegetarians consumed vegetables significantly more often than the omnivores, the intake (32 times month ${ }^{-1}$ ) was not as often as might be expected of a vegetarian popula- tion. Furthermore, there was no difference between the female groups in the frequency of consumption of, for example, fruits/berries, ice cream and sweets/chocolates. Male vegetarians showed no major differences in food intake compared with male omnivores, except for eating animal products, margarine, ice cream, cake/cookies, and fried and barbecued food less often. The male vegetarians reported eating vegetables less than once a day $(25$ times month ${ }^{-1}$ ). Furthermore, lifestyle characteristics of vegetarians were similar to those of omnivores regarding exercise, use of alcohol and smoking habits (3). These findings indicate that the young vegetarians in the present study have different food habits and lifestyles compared with findings from previous studies of vegetarians, and that adherence to a low-meat diet may not correlate with other health-promoting practices among adolescents in Sweden and Norway.

The nutrient intake and status of 30 vegans (16-20 years old) and 30 omnivores matched by gender, age and height was investigated by the diet history method and blood samples (5). The diet history was validated by a doubly labelled water method for reported energy intake of 32 subjects, and by four $24 \mathrm{~h}$ urine collections for reported nitrogen (protein), sodium and potassium of 60 subjects $(4,5)$. The diet history interview had a bias towards underestimating the energy intake (mean $-1.93 \mathrm{MJ}$ day $^{-1}, 96 \%$ confidence interval -2.89 to $-0.97 \mathrm{MJ} \mathrm{day}^{-1}$ ) (5). However, no significant difference in validity of reported energy and protein intakes was found between vegans and omnivores, which enabled comparison between the dietary groups.

Vegans as well as omnivores are in general two extremely heterogeneous groups of people with regard to dietary intake. The groups were also heterogeneous in this study, but some common features could be seen, such as positive aspects of the vegan diet, which were a lower content of total fat, cholesterol and protein, a higher content of carbohydrates $(\mathrm{E} \%)$, fibre, vitamin $\mathrm{C}$, vitamin $\mathrm{E}$ 
and magnesium, and a better fatty acid composition (5). The negative aspects were a lower content of vitamin $B_{12}$, vitamin $D$, calcium and selenium. A significantly larger number of female vegans had a total intake of vitamin $\mathrm{B}_{12}(n=8)$, vitamin $\mathrm{D}(n=7)$ and calcium $(n=10)$ below average requirements. The vegan females $(n=11)$ also had too low an intake of selenium and $79 \%$ of the average requirement was obtained from diet and supplements. The selenium intake from diet alone was below the average requirement for 11 female omnivores, but intakes of all other nutrients were above average requirements at group level.

A significantly larger number of male vegans had a lower dietary intake than average requirements for riboflavin $(n=11)$ and vitamin $\mathrm{B}_{12}(n=15)$, but the number decreased if intake from dietary supplements was included (5). Calcium and selenium intakes were below average requirements for vegan males ( $n=10$ and 11, respectively), even when intake from dietary supplements was included. Only $65 \%$ and $98 \%$, respectively, of the selenium and calcium average requirements were obtained from diet and supplements, respectively. The male omnivores had an intake above average requirements of all nutrients, except for one male who had a lower intake (including supplements) of selenium compared with average requirement.

Low iron stores were found only among female subjects and were as prevalent among vegans as among omnivores (20\% and $23 \%$, respectively) (5). Female vegans had a higher haemoglobin concentration than female omnivores, but there was no difference in the S-ferritin level. Three out of 30 vegans had serum vitamin $B_{12}$ concentrations lower than the reference range.

Vegetarianism can be seen as either a dietary fad, an ethical choice or a political statement. Therefore, eating vegetarian food may not necessarily mean having a healthy diet, just as eating omnivorous food may not always be adequate for all nutrients. Adequate nutrition depends on what foods and amounts are included in the diet. It is important for adolescents in general and vegetarians in particular to receive more knowledge, both theoretical and practical, about how to combine and prepare a healthy diet. Schools and parents have an important role to play by increasing knowledge and making well-planned foods available.

Papers $(1-5)$ in this thesis provide information which indicates that the new generation of vegetarians has different dietary and lifestyle habits to those shown by previous studies of vegetarians. For vegetarian adolescents, especially vegans, it is essential to be well motivated, to take an active interest in the new dietary regimen and to appreciate that becoming vegetarian is not simply a matter of excluding certain food items. Excluded animal products should be replaced with equally nutritious vegetarian products. If there is no interest in nutrition and no nutritious vegetarian food available at home or in school, a poor eating pattern may be the consequence. Some schools choose not to serve a vegan lunch alternative, for various reasons, but vegans will be unlikely to eat animal products just because vegan food is not served for school lunch. Rather, the vegan will probably eat a less nutritious lunch or nothing at all. It is possible for young vegans to have an adequate nutrient intake, but vitamin $B_{12}$ supplements are needed and care needs to be taken to ensure sufficient intake of some nutrients, especially selenium and calcium.

There is a need for future research into the long-term health effects of being vegetarian. The vegans in the present thesis showed a lower intake of calcium, and the energy expenditure indicated less physical activity compared with omnivores, which may result in a lower bone mineral density. There should also be a high priority to monitor the prevalence of vitamin $B_{12}$ deficiency in long-term vegetarians.

\section{Articles in the Thesis}

1. Larsson C, Johansson G. Prevalence of vegetarians in Swedish secondary schools. Scand J Nutr 1997;41:11720.

2. Larsson C, Klock K, Nordrehaug Åstrøm A, Haugejorden O, Johansson G. Food habits of young Swedish and Norwegian vegetarians and omnivores. Public Health Nutr 2001;4:1005-14.

3. Larsson C, Klock K, Nordrehaug Åstrøm A, Haugejorden O, Johansson G. Lifestyle-related characteristics of young low-meat consumers and omnivores in Sweden and Norway. J Adolesc Health 2002 (in press).

4. Larsson C, Westerterp K, Johansson G. Validity of reported energy expenditure and energy and protein intakes of Swedish vegan and omnivore adolescents. Am J Clin Nutr 2002;75:268-74.

5. Larsson C, Johansson G. The dietary intake and nutritional status of young vegans and omnivores in Sweden. Am J Clin Nutr 2002. (accepted for publication).

The article is based on the author's thesis, which was conducted at the Department of Food and Nutrition, Umeå University, Sweden. Defended on December 7, 200।. ISBN: 9|-7|9|-983-X

Christel Larsson, PhD, Nutritionist

E-mail: christel.larsson@kost.umu.se 